\title{
A brief computerised discharge summary in old age psychiatry: general practitioner reactions
}

\author{
J. P. WAtris, Senior Lecturer/Consultant in the Psychiatry of Old Age, \\ St James's University Hospital; and DAVID PROTHEROE, \\ Senior House Officer in Psychiatry, St James's University Hospital, Leeds LS9 7TF
}

In an attempt to provide data for medical audit, to standardise the information on discharge letters and to speed communication, a computerised discharge form was introduced for a catchment area of 20,000 people over 65 years. The programme, based on a surgical audit programme using DBASE II, was developed by one of us to run on an Amstrad PCW. This produced a discharge summary on a standard layout which gave the date of admission, the patient's name, date of birth and address as well as the consultant and GP's name. The patient's diagnosis (according to ICD-9), and a list of disciplines and facilities involved in follow-up preceded brief notes on history and progress. Date of discharge was followed by a list of medication and a space for 'other information'. All this was contained on one side of A4 paper.

The Health Authority were sufficiently impressed to find an upgraded version of the system designed to cover community and day patients. In order to inform developments, GPs' views were sought.

\section{Findings}

The survey was based on 50 consecutive elderly psychiatric patients discharged from St James's University Hospital and Seacroft Hospital in Leeds. We sent short questionnaires to all 43 GPs who had received one or more discharge summaries.

Out of 43 GPs, 28 responded (65\%). Between 26 and 28 GPs answered each question, and percentages have been used to simplify the following report; $81 \%$ of GPs said that they preferred the new format.

On diagnosis, all GPs thought the information given was about right. Information on follow-up was thought sufficient by $70 \%$ although $30 \%$ wanted more. The information on history and progress was thought about right by $73 \%$ but $23 \%$ wanted more.
A shorter summary of 1-2 sides of A4 with the opportunity to write or phone for more was preferred by $93 \%$.

Of GPs, $44 \%$ ideally wished to receive the discharge summary 4-7 days after discharge; $37 \%$ preferred 2-3 days; and $19 \%$ within a day of discharge.

Only $7 \%$ of GPs thought the summary should be sent with the patient or relative. First class post was preferred by $56 \%$ and $19 \%$ second class post. Electronic mail such as the Merlin Health Net System was preferred by $19 \% ; 11 \%$ of GPs had facilities for electronic mail but a further $11 \%$ were planning to get them.

GPs were invited for their comments. A note of information given to patients and relatives was wanted by $15 \%$. Other comments were varied and generally favourable.

\section{Comment}

General practitioners generally preferred a short standard format discharge summary. This is consistent with the findings of Craddock \& Craddock (1989). Some information potentially of use to the psychiatrist is lost but this should be present in the written case notes and could be provided as a separate written summary. The standardised summary has two additional advantages. Firstly it can potentially be sent very quickly. Secondly it accumulates information on a computer database in a way that enables analysis of workload and facilitates audit.

\section{Reference}

Craddock, N. \& Craddock, B. (1989) Psychiatric discharge summaries: differing requirements of psychiatrists and general practitioners. British Medical Journal, 299, 1382. 